\title{
Dodging COVID-19 infection: Low expression and localization of Angiotensin-Converting Enzyme 2 (ACE2) and Transmembrane Serine Protease 2 (TMPRSS2) in Mesenchymal Stem Cells derived from human umbilical cord (hUC-MSCs).
}

Jonathan J Hernandez ( $\square$ jhernandez@aidanresearch.com )

Aidan Research and Consulting LLC/ Medistem Panama https://orcid.org/0000-0003-3319-5468

Doyle E Beaty

Aidan Research and Consulting LLC

Logan L Fruhwirth

Aidan Research and Consulting LLC

J M Sloan

Aidan Research and Consulting LLC

Ana P Lopes Chaves

Aidan Research and Consulting LLC

Neil H Riordan

Aidan Research and Consulting LLC

\section{Research Article}

Keywords: COVID-19, ACE2, TMPRSS2, hUC-MSCs, SARS-CoV-2

Posted Date: June 19th, 2020

DOI: https://doi.org/10.21203/rs.3.rs-36376/v1

License: (c) (i) This work is licensed under a Creative Commons Attribution 4.0 International License.

Read Full License 


\section{Abstract}

Mesenchymal stem cells derived from human umbilical cord (hUC-MSCs) have immunomodulatory properties that are of interest to treat novel coronavirus disease 2019 (COVID-19). Leng et al. recently reported that hUC-MSCs derived from one donor negatively expressed Angiotensin-Converting Enzyme 2 (ACE2), a key protein for viral infection along with Transmembrane Serine Protease 2 (TMPRSS2). In this report, the expression of ACE2 and TMPRSS2 was analyzed in 24 lots of hUC-MSCs derived from 24 different donors via quantitative polymerase chain reaction (qPCR), Western Blot, immunofluorescence and flow cytometry. hUC-MSCs had significantly lower ACE2 $(p=0.002)$ and TMPRSS2 $(p=0.008)$ expression compared with human lung tissue homogenates in Western blot analyses. Little to no expression of ACE2 or TMPRSS2 was observed in hUC-MSC by QPCR, and they were not observable with immunofluorescence in hUC-MSCs cell membranes. A double negative ACE2 and TMPRSS2 population percentage of $94.30 \% \pm 15.55$ was obtained for hUC-MSCs via flow cytometry, with only $0.011 \%$ ACE2 and 10.91\% TMPRSS2 observable positive populations. We have demonstrated negative expression of ACE2 and low expression of TMPRSS2 in 24 lots of hUC-MSCs. This has crucial implications for the design of future therapeutic options for COVID-19, since hUC-MSCs would have the ability to "dodge" viral infection to exert their immunomodulatory effects.

\section{Introduction}

With the worsening of the public health emergency caused by the global spread of the novel coronavirus disease 2019 (COVID-19) comes a pressing need to understand the molecular mechanisms driving the infection within the body ${ }^{1}$. The spike glycoprotein of SARS-CoV-2, the virus responsible for COVID-19, allows for entry into cells via human angiotensin converting enzyme II (ACE2) once primed by the cellular serine protease TMPRSS2 ${ }^{2-4}$. Therefore, cell membrane ACE2 and TMPRSS2 are integral components of viral transmission and spread ${ }^{2}$.

A hyperinduction of inflammatory cytokines and chemokines such as interleukin (IL)-2R, IL-6, IL-8, IL-10, and tumor necrosis factor (TNF)- $a$ among others, occurs in the lungs of critical patients with COVID-19 pneumonia: a "cytokine storm" ${ }^{5-7}$. Cytokine storms can result in pulmonary edema, air exchange dysfunction, acute respiratory distress syndrome, acute heart failure, secondary infections, and death ${ }^{8}$. In light of current treatments for severe cases of COVID-19 having had mixed results or serious adverse events $^{9-11}$, there remains a global interest to find safe and effective avenues of treatment.

Mesenchymal stem cells (MSCs) ${ }^{12,13}$ exert anti-inflammatory ${ }^{14-16}$, anti-bacterial, anti-protozoan and antiviral ${ }^{17-20}$ effects making them a possible treatment for COVID-19-related complications ${ }^{21-24}$. MSCs produce several types of cytokines via paracrine secretion or have direct interactions with immune cells, resulting in immunomodulation ${ }^{25}$. MSCs derived from the Wharton Jelly tissue of the human umbilical cord (hUC-MSCs) possess a high capacity for proliferation and immunomodulation ${ }^{26-28}$. In a study of 150 people with confirmed cases of COVID-19, levels of the inflammatory cytokine IL- 6 were significantly 
higher in non-survivors than in survivors of the disease ${ }^{29}$. Furthermore, the upregulation of IL-6 in fatal cases of COVID-19 indicates that mortality may be driven by hyperinflammation ${ }^{30}$. UC-MSCs have been used to modulate IL- 6 in the body: for example, the levels of IL- 6 decreased around $50 \%$ three months after treatment in a study of 172 patients with rheumatoid arthritis ${ }^{31}$.

hUC-MSCs were used experimentally to treat seven patients with confirmed COVID-19 pneumonia in China $^{32}$. The pulmonary function of all patients significantly improved within two days of hUC-MSC transplantation and levels of TNF-a were significantly reduced. Additionally, the gene expression profile in this study showed that MSCs were ACE2- and TMPRSS2-. However, the hUC-MSCs used for treatment were derived from only one donor, representing a limitation for a broader extrapolation of these results.

In this study, we investigated the expression of ACE2 and TMPRSS2 in hUC-MSCs lines derived from different donors using quantitative polymerase chain reaction (qPCR), Western Blot, immunofluorescence and flow cytometry. Human pulmonary alveolar cells type I, human lung homogenates, human lung RNA, and one lot of hUC-MSCs transfected with an ACE2 expression plasmid were used as controls.

\section{Materials And Methods}

\section{- Cell Culture}

Human pulmonary alveolar epithelial cells type I were obtained from AcceGen Biotechnology (Cat.\# ABCTC3770) and cultured following the manufacturer's guidelines.

Twenty-four lots of culture expanded human hUC-MSCs were utilized in this study, isolated from human umbilical cord tissue from normal, healthy births, voluntarily donated with a fully executed informed consent form. Sixteen of those lots were obtained from a biotechnology company that manufactures hUC-MSCs for use in clinical trials (Medistem Panama, City of Knowledge of the Republic of Panama). The cell lots were passage 5 and were received frozen in dry shippers and stored at $-150^{\circ} \mathrm{C}$ until studied. Manufacturing methodology is described in detail in publications of clinical trials that used these hUCMSCs for treatment. ${ }^{33,34}$

For the other eight lots, umbilical cords were obtained from a tissue acquisition company licensed by the American Association of Tissue Banks (AATB) in the United States. The isolation, selection and culture were performed by Aidan Research and Consulting LLC for research purposes only. The isolation process was done using the Umbilical Cord Dissociation Kit, human (Miltenyi Biotec 130-105-737) following the manufacturer's guidelines. When colony-forming units reached $70 \%$ of confluency, hUC-MSCs were selected using the MSC Phenotyping Kit, human (Miltenyi Biotec 130-125-285) and $>95 \%$ positive cells for CD73/CD105 were sorted using SH800 cell sorter (Sony Biotech). Cells were expanded through passage 5 and used for the measurements reported here.

All cell lots used in this study met release criteria, namely: $75 \%$ viability and $>95 \%$ positive for CD 90 , CD73, CD105 cell surface markers as determined by flow cytometry. These 24 lots were all used as 
samples for the subsequent experiments, and one of the Aidan Research and Consulting lots was transfected with an ACE-2 plasmid and used as a positive control for ACE-2 measurements.

\section{- Transient Transfection}

For transfection, 6,000 hUC-MSCs per $\mathrm{cm}^{2}$ were plated in $100 \mathrm{~cm}^{2}$ cell culture dishes. Once they reached $70 \%$ of confluency, $3 \mu \mathrm{g}$ of ACE2 plasmid was transfected using Lipofectamine ${ }^{\mathrm{TM}}$ Stem Transfection Reagent (Sino Biological HG10108-ACR) following the manufacturer's instructions. Twenty-four hours after transfection cells were either fixated using Image-i $T^{\mathrm{TM}}$ Fixation/Permeabilization Kit (Thermo Fisher Scientific \#R37602) for imaging or lysed using RIPA buffer (Thermo Fisher Scientific \#89901) with 1X protease and phosphatase inhibitor (Thermo Fisher Scientific 78444) for Western Blot analysis.

\section{- Protein preparation and Western blot}

Human lung homogenates were purchased from the OriGene tissue bank (CP565585, CP565542, CP565577, CP565443 and CP565542). Whole protein was obtained by sonication of a pellet of harvested hUC-MSCs and quantified using the Pierce ${ }^{\text {TM }}$ Rapid Gold BCA Protein Assay Kit (Thermo Fisher Scientific \#A53226). $25 \mu \mathrm{g}$ of protein was added to $4 x$ LDS loading buffer and incubated at $50^{\circ} \mathrm{C}$ for 5 minutes. SDS-PAGE was performed with Criterion TGX Stain-free 4-20\% Gel (Bio-Rad \#5678093) and transferred to a PVDF membrane using the iBlot ${ }^{\mathrm{TM}} 2 \mathrm{Gel}$ Transfer Device (Thermo Fisher Scientific \#IB21001). Membranes were blocked for 1 hour in Pierce Clear Milk Blocking Buffer (Thermo Fisher Scientific \# 37587) at room temperature and incubated overnight at $4^{\circ} \mathrm{C}$ with 1:500 Rabbit anti-ACE2 (Invitrogen, MA5-32307), 1:500 primary antibody TMPRSS2 made in rabbit (Abcam \# ab92323), and 1 hour at room temperature with 1:10,000 Mouse anti-GAPDH (Millipore, MAB374). Membranes were incubated in secondary antibodies, 1:5,000 Alexa Fluor 680 goat anti-rabbit (Life Technologies, A21076) and 1:5,000 Alexa Fluor 488 donkey anti-mouse (Invitrogen: A21202), for one hour at room temperature. Detection of relevant proteins and images were taken using iBright FL1500 Imaging System (Invitrogen). For relative quantification, the volume intensity of the bands was obtained using iBright software. The relative expression was calculated by dividing the values to GAPDH.

\section{- Quantitative real-time Polymerase chain reaction (qPCR)}

Total RNA was isolated from cells using the Trizol ${ }^{\text {TM }}$ Plus RNA Purification Kit (Invitrogen [Thermo Fisher Scientific]) and DNA was removed using the TURBO DNA-free Kit (Invitrogen) from all hUC-MSC lots. Additionally, RNA isolated from human lung tissues (OriGene Technologies; cat.\#: CR559346, CR559185, CR560789, CR562469 and CR561266) was included as a positive control $(n=5)$. All RNA extractions were then quantified using a Varioskan LUX ${ }^{\mathrm{TM}}$ (Thermo Fisher), and their integrity was checked using a $1 \% \mathrm{E}-$ $\mathrm{Gel}^{\mathrm{Tm}}$ EX Agarose Gel (Thermo Fisher Scientific). Subsequently, $200 \mathrm{ng}$ of purified RNA was reversetranscribed to $c D N A$ using the iScript ${ }^{\mathrm{TM}} \mathrm{cDNA}$ Synthesis Kit (Biorad) following the manufacturer's protocol. Then, 20 ng of cDNA was amplified by qPCR using the TaqMan ${ }^{\text {TM }}$ Fast Advanced Master Mix along with TaqMan $^{\text {TM }}$ Gene Expression Assays for ACE2 (Hs01085333_m1), TMPRSS2 (Hs01120965_m1), and for the reference gene PPIA (Hs99999904_m1). All qPCR reactions were performed in triplicates on a 
QuantStudio $^{\text {TM }} 3$ Real-Time PCR System (Applied Biosystems). Raw cycle thresholds values were calculated using the QuantStudio ${ }^{\mathrm{TM}}$ Design and Analysis Software v.1.5.1 using automatic baseline settings and a threshold of 0.3 . The relative expression of genes of interest was normalized to the expression of PPIA. A Mann-Whitney Rank Sum Test was used to calculate statistically significant differences between expression of ACE2 and TMPRSS2 in human lung RNA, human pulmonary alveolar type I cells, and UC-MSCs.

\section{- Immunofluorescence}

Cells were plated on 12-well glass-bottomed MatTek plates (P12G-1.5-14-F) and fixed using an Image-iT ${ }^{\mathrm{TM}}$ Fixation/Permeabilization Kit (Thermo Fisher Scientific \#R37602) following the manufacturer's instructions. Primary antibody ACE2 anti-rabbit (Invitrogen: MA5-32307), in a dilution 1:50, was added to the indicated wells and left overnight at $4^{\circ} \mathrm{C}$. The primary antibody TMPRSS2 made in rabbit (Abcam \# ab92323), in a dilution 1:50 was used and incubated at room temperature for 2 hours. Secondary antibodies Alexa Fluor 488 donkey anti-mouse (Invitrogen A21202) and Cyanine3 goat anti-rabbit (Invitrogen: A10520), in a dilution 1:50, were added to the wells and incubated for one hour at room temperature. Coverslips were mounted onto the slides using Prolong DAPI (Invitrogen P36935) and photographs were taken using a Lionheart FX automated microscope (BioTek).

\section{- Flow Cytometry}

Single cell suspensions of 24 hUC-MSC lots and one alveolar cell line were stained with primary antibody TMPRSS2 made in rabbit (Abcam, ab92323) and with a secondary PE-Cy5.5 goat anti-rabbit antibody (Invitrogen, L42018). Afterwards, the cells were stained with a conjugated FITC anti-human ACE2 antibody made in rabbit (LSBio, LS-C458088). Cells from 5 Medistem lots were stained with the TMPRSS2 secondary antibody to show Non-Specific-Binding (NSB). The cells were resuspended in 300 $\mu \mathrm{L}$ of sorting buffer ( $0.05 \%$ FBS in PBS) and analyzed using the flow cytometry functions on a Sony SH800S Cell Sorter.

\section{- Statistical analysis}

SigmaPlot 12.5 (Systat Software) was used for all statistical analyses. Means and standard errors of the means were calculated for all relevant quantities. One-way ANOVA (Mann-Whitney Rank Sum Test) was used for all comparisons. A p-value $\leq 0.05$ was taken as the level for statistical significance.

\section{Results}

- Western blot reveals little expression of ACE2and low levels of TMPRSS2 and in hUC-MSCs cell lysates.

hUC-MSCs $(n=24)$ had significantly lower $(p=0.002)$ ACE2 expression relative to GAPDH compared with lung tissue homogenates $(n=4)$ in Western blot analyses (Figure 1). A trend for lower expression of ACE2 
in hUC-MSCs was observed when compared to hUC-MSCs transfected with ACE2 expressing plasmid $(n=1)$. Alveolar cells $(n=1)$ also showed low expression of ACE2. TMPRSS2 levels in hUC-MSCs were significantly lower when compared to lung tissue $(p=0.008)$. Although expression was low, TMPRSS2 levels were variable between hUC-MSCs from different donors. ACE2 bands were observed to have a molecular weight of $120 \mathrm{kDa}$, while TMPRSS2 showed a thin band around $50 \mathrm{kDa}$. Expression of both proteins was normalized to GAPDH, which was observed at $37 \mathrm{kDa}$.

\section{- Low gene expression of ACE2 and TMPRSS2}

Expression of ACE2 and TMPRSS2 was observed in all human lung RNA samples $(n=5)$ while little to no expression was observed in hUC-MSC $(n=24)$, or in human pulmonary alveolar type I cells $(n=1)$ observed on qPCR. Alveolar cells expressed less TMPRSS2 than the lung tissue, and did not express ACE2. For each sample, relative expression of the genes was averaged to represent differences between the sample groups. Significant differences in the expression of ACE2 and TMPRSS2 were observed between the human lung RNA and hUC-MSCs ( $p \leq 0.001$ for both) according to the Mann-Whitney Rank Sum Test (Figure 2). Overall, expression of TMPRSS2 was higher in all groups.

\section{- ACE2 and TMPRSS2 are not localized in the cell membrane of hUC-MSCs}

Fluorescence microscopy was conducted to determine the presence and localization of ACE2 and TMPRSS2 in the membrane of the cells (human pulmonary alveolar cells type I, hUC-MSC transfected with an ACE2 plasmid, and hUC-MSCs). ACE2 and TMPRSS2 were not observable in the cell membrane of hUC-MSCs (Figure 3). However, the expression of TMPRSS2 was observed intra-cellularly in all the groups. hUC-MSCs transfected with an ACE2 plasmid restored the expression of ACE2 in the cell membrane (Figure 3).

\section{- Most hUC-MSCs populations are doubly negative for ACE2 and TMPRSS2.}

Flow cytometry of cell populations of hUC-MSC and human pulmonary alveolar cells type I were gated into four quadrants, as well as hUC-MSC with non-specific binding (hUC-MSC-NSB) to discard false positive cell populations from the test group (Figure 4). The hUC-MSC population percentages $(n=24)$ were $0.011 \% \pm 0.038$ ACE2 positive, $5.30 \% \pm 2.85$ TMPRSS2 positive, $0.274 \% \pm 0.087$ double positive, and $88.69 \pm 15.48$ double negative. The NSB population percentages $(n=5)$ were $0.002 \% \pm 0.00082$ ACE2 positive, $5.61 \% \pm 1.54$ TMPRSS2 positive, $0.0067 \% \pm 0.0022$ double positive, and $94.38 \pm 7.88$ double negative. The alveolar population percentages $(n=1)$ were $3.909 \%$ ACE2 positive, $1.87 \%$ TMPRSS2 positive, $1.368 \%$ double positive, and $92.85 \%$ double negative. A true TMPRSS2 positive population percentage of $10.91 \% \pm 2.40$ was obtained for the hUC-MSC population after removing the $5.61 \%$ TMPRSS2 NSB percentage. Similarly, the true double negative ACE2 population percentage was $94.30 \%$ \pm 15.55 .

\section{Discussion}


Mesenchymal stem cells (MSCs) are currently being considered as a potential treatment of COVID-19 and its associated complications ${ }^{22,23}$. Given that ACE2 and TMPRSS2 are crucially involved in transmission and spread of the virus, we sought to investigate their expression in hUC-MSCs. In this study, we have demonstrated the negative expression of ACE2 and low expression of TMPRSS2 in 24 lots of hUC-MSCs derived from different donors. We investigated levels of gene expression and localization of the protein in the cells via different methods: gene expression was not found using qPCR and Western Blot in any of the 24 lots of hUC-MSCs compared to the positive control of human lung RNA; ACE2 was not detected in the hUC-MSCs membrane with immunofluorescence, and flow cytometry revealed that only $0.3 \%$ of MSCs positively expressed ACE2 and TMPRSS2. This broadens the novel findings of Leng et al. regarding the expression of these specific genes in hUC-MSCs ${ }^{32}$ to a larger number of cell lots derived from different donors.

Lower expression or lack of expression of ACE2 and TMPRSS2 could have crucial implications for the design of future therapeutic options for COVID-19. Since the virus engages ACE2 as the entry receptor ${ }^{35}$ and employs the cellular serine protease TMPRSS2 for the spike glycoprotein S priming ${ }^{36,37}$, ACE2- and low TMPRSS2 expressing cells should likely remain uninfected by the virus (Figure 5 ). Demonstrating that hUC-MSCs do not appreciatively express ACE2 and express low levels of TMPRSS2, coupled with their immunomodulatory, anti-inflammatory and antimicrobial properties, could position them as a viable treatment option. After being administered to a COVID-19 patient, a large majority of hUC-MSCs should be able to exert their therapeutic action via the secretion of anti-inflammatory molecules while "dodging" the virus (Figure 5). To date, MSCs have been used to treat pulmonary conditions such as idiopathic pulmonary fibrosis, acute respiratory distress syndrome and chronic obstructive pulmonary disease ${ }^{14}$. MSC-derived exosomes were also able to revert pulmonary fibrosis in a mouse model ${ }^{38}$. Likewise, trophic factors secreted by MSCs (MTF), which can be administered by inhalation, have shown therapeutic benefits for pulmonary disease ${ }^{39,40}$. In light of this, international trials are currently ongoing to treat COVID-19 with mesenchymal stem cells derived from a variety of tissues ${ }^{41}$.

The intent of investigating the expression of ACE2 and TMPRSS2 was to determine the likelihood of hUCMSCs becoming infected by SARs-Cov-2, as it would be counterproductive to supply the virus with a fresh influx of susceptible cells. Quality control is of utmost importance when considering treatment with MSCs for COVID-19; in addition to investigating the infectivity potential of MSCs, attention should also be given to concerns about the variability of TF/CD142 expression among cells lots, which may trigger blood clotting and thromboembolism in this hypercoagulable pathology ${ }^{42}$. High expression of TF/CD142 on cells used in the treatment of Covid-19 could lead to dire consequences if the cells enhanced the already present pro-thrombotic effects of the viral infection itself 43,44 .

The extent of the ability of hUC-MSCs to mitigate the devastating cytokine storm observed in critically ill patients should be a next avenue in further research, particularly to ascertain which cytokines can be induced back to their normal levels in the presence of hUC-MSC secretions - this could lead to more targeted therapeutics. Since the levels of inflammatory cytokines and chemokines have been reported to 
be abnormally elevated in critically ill COVID-19 patients ${ }^{5,32}$, the immunomodulatory effects of hUC-MSCs on these cytokines, and particularly in a setting of simultaneous overexpression, should be investigated promptly. Caution should be exerted, however, to administer hUC-MSCs outside of the clinically demonstrable cytokine storm, as MSC immunomodulatory effects could paradoxically aid the virus in earlier phases of the infection.

Furthermore, AGTR2 could be a third marker of interest in addition to ACE2 and TMPRSS2, since its affinity to the spike protein of the virus appears to be higher than ACE $2^{45}$. Over the course of this study, we began investigating AGTR2 expression in hUC-MSCs compared to controls, finding negative expression (data not shown). We hope to expand upon these findings in future experiments.

Multiple repositories were contacted at the beginning of this study and alveolar cells type II were not available to us at the time, resulting in significant limitations for this study. The impossibility to obtain said alveolar cells for research in a reasonable timeframe meant choosing human lung and as pulmonary alveolar cells type I as controls in limited quantities. Alveolar cells type II would have been more ideal to replicate the more intrinsic mechanisms of SARS-CoV-2 infection, but we hope that these results may be extended to that type of cell when it becomes more readily available for research. Only twenty-four different donors were considered for this experiment, but we expect that these results could be replicated with a larger number of hUC-MSC lots. Finally, our current laboratory does not possess BSL-4 facilities that would allow culturing and testing the infection rate of SARS-CoV-2 in hUC-MSCs to verify our findings.

\section{Conclusions}

hUC-MSCs have the potential to provide a safe, effective treatment for critically ill COVID-19 patients, which could help mitigate the devastating economic and public health consequences caused by the rapid worldwide spread of SARS-CoV-2. We have demonstrated negative expression of ACE2 and low expression of TMPRSS2, key proteins in the SARS-Cov2 infection process, in twenty-four lots of hUCMSCs, and we hope that these results will encourage further research into hUC-MSCs for the treatment of the inflammatory effects of COVID-19 infection.

\section{Declarations}

\section{Acknowledgments}

We would like to thank Ms. Avila for her help in the preparation of this manuscript, and Brenda Mendoza Flores for her assistance with immunofluorescence images.

\section{Author Contributions}

All authors have contributed equally to this manuscript. 


\section{Conflict of Interests}

NHR is a shareholder and CEO of Aidan Research and Consulting LLC. All other authors declare no conflicts of interest.

\section{Data Availability}

The data that support the findings of this study are available from the corresponding author upon reasonable request.

\section{References}

1. World Health Organization. Coronavirus disease (COVID-2019) situation reports. https://www.who.int/emergencies/diseases/novel-coronavirus-2019/situation-reports. Accessed June, 2020.

2. Hoffmann M, Kleine-Weber H, Schroeder S, et al. SARS-CoV-2 Cell Entry Depends on ACE2 and TMPRSS2 and Is Blocked by a Clinically Proven Protease Inhibitor. Cell. 2020.

3. Ge XY, Li JL, Yang XL, et al. Isolation and characterization of a bat SARS-like coronavirus that uses the ACE2 receptor. Nature. 2013;503(7477):535-538.

4. Kuba K, Imai Y, Rao S, et al. A crucial role of angiotensin converting enzyme 2 (ACE2) in SARS coronavirus-induced lung injury. Nat Med. 2005;11(8):875-879.

5. Qin C, Zhou L, Hu Z, et al. Dysregulation of immune response in patients with COVID-19 in Wuhan, China. Clin Infect Dis. 2020.

6. Liu Q, Zhou Y-h, Yang Z-q. The cytokine storm of severe influenza and development of immunomodulatory therapy. Cellular \& Molecular Immunology. 2016;13(1):3-10.

7. Huang C, Wang Y, Li X, et al. Clinical features of patients infected with 2019 novel coronavirus in Wuhan, China. Lancet. 2020;395(10223):497-506.

8. Metcalfe SM. Mesenchymal stem cells and management of COVID-19 pneumonia. Medicine in Drug Discovery. 2020;5:100019.

9. Yazdany J, Kim AHJ. Use of Hydroxychloroquine and Chloroquine During the COVID-19 Pandemic: What Every Clinician Should Know. Ann Intern Med. 2020.

10. Cao B, Wang Y, Wen D, et al. A Trial of Lopinavir-Ritonavir in Adults Hospitalized with Severe Covid19. N Engl J Med. 2020.

11. Cortegiani A, Ingoglia G, Ippolito M, Giarratano A, Einav S. A systematic review on the efficacy and safety of chloroquine for the treatment of COVID-19. J Crit Care. 2020.

12. Bonfield TL, Caplan Al. Adult mesenchymal stem cells: an innovative therapeutic for lung diseases. Discov Med. 2010;9(47):337-345.

13. Caplan Al. Mesenchymal stem cells. J Orthop Res. 1991;9(5):641-650. 
14. Antoniou KM, Karagiannis K, Tsitoura E, et al. Clinical applications of mesenchymal stem cells in chronic lung diseases. Biomed Rep. 2018;8(4):314-318.

15. Regulski MJ. Mesenchymal Stem Cells: "Guardians of Inflammation". Wounds : a compendium of clinical research and practice. 2017;29(1):20-27.

16. Zachar L, Bacenkova D, Rosocha J. Activation, homing, and role of the mesenchymal stem cells in the inflammatory environment. Journal of inflammation research. 2016;9:231-240.

17. Meisel R, Brockers S, Heseler K, et al. Human but not murine multipotent mesenchymal stromal cells exhibit broad-spectrum antimicrobial effector function mediated by indoleamine 2,3-dioxygenase. Leukemia. 2011;25(4):648-654.

18. Laffey JG, Matthay MA. Fifty Years of Research in ARDS. Cell-based Therapy for Acute Respiratory Distress Syndrome. Biology and Potential Therapeutic Value. Am J Respir Crit Care Med. 2017;196(3):266-273.

19. Qian X, Xu C, Fang S, et al. Exosomal MicroRNAs Derived From Umbilical Mesenchymal Stem Cells Inhibit Hepatitis C Virus Infection. Stem Cells Trans/ Med. 2016;5(9):1190-1203.

20. Monsarrat $P$, Kemoun $P$, Casteilla $L$, Planat-Benard V. Broad-Spectrum Antibacterial Effects of Human Adipose-Derived Stromal Cells. Stem Cells Int. 2019;2019:5389629.

21. Shetty AK. Mesenchymal Stem Cell Infusion Shows Promise for Combating Coronavirus (COVID-19)Induced Pneumonia. Aging Dis. 2020;11(2):462-464.

22. Bari E, Ferrarotti I, Saracino L, Perteghella S, Torre ML, Corsico AG. Mesenchymal Stromal Cell Secretome for Severe COVID-19 Infections: Premises for the Therapeutic Use. Cells. 2020;9(4).

23. Atluri S, Manchikanti L, Hirsch JA. Expanded Umbilical Cord Mesenchymal Stem Cells (UC-MSCs) as a Therapeutic Strategy in Managing Critically III COVID-19 Patients: The Case for Compassionate Use. Pain Physician. 2020;23(2):E71-E83.

24. Khoury M, Cuenca J, Cruz FF, Figueroa FE, Rocco PRM, Weiss DJ. Current Status of Cell-Based Therapies for Respiratory Virus Infections: Applicability to COVID-19. Eur Respir J. 2020.

25. Kyurkchiev D, Bochev I, Ivanova-Todorova E, et al. Secretion of immunoregulatory cytokines by mesenchymal stem cells. World J Stem Cells. 2014;6(5):552-570.

26. Madrigal M, Rao KS, Riordan NH. A review of therapeutic effects of mesenchymal stem cell secretions and induction of secretory modification by different culture methods. Journal of translational medicine. 2014;12(1):260.

27. Nagamura-Inoue T, He H. Umbilical cord-derived mesenchymal stem cells: Their advantages and potential clinical utility. World J Stem Cells. 2014;6(2):195-202.

28. Weiss ML, Anderson C, Medicetty S, et al. Immune properties of human umbilical cord Wharton's jellyderived cells. Stem cells. 2008;26(11):2865-2874.

29. Ruan Q, Yang K, Wang W, Jiang L, Song J. Clinical predictors of mortality due to COVID-19 based on an analysis of data of 150 patients from Wuhan, China. Intensive Care Med. 2020. 
30. Mehta P, McAuley DF, Brown M, et al. COVID-19: consider cytokine storm syndromes and immunosuppression. Lancet. 2020;395(10229):1033-1034.

31. Wang $L$, Wang $L$, Cong $X$, et al. Human umbilical cord mesenchymal stem cell therapy for patients with active rheumatoid arthritis: safety and efficacy. Stem Cells Dev. 2013;22(24):3192-3202.

32. Leng Z, Zhu R, Hou W, et al. Transplantation of ACE2(-) Mesenchymal Stem Cells Improves the Outcome of Patients with COVID-19 Pneumonia. Aging Dis. 2020;11(2):216-228.

33. Riordan NH, Hincapie ML, Morales I, et al. Allogeneic Human Umbilical Cord Mesenchymal Stem Cells for the Treatment of Autism Spectrum Disorder in Children: Safety Profile and Effect on Cytokine Levels. Stem cells translational medicine. 2019;8(10):1008-1016.

34. Riordan NH, Morales I, Fernandez G, et al. Clinical feasibility of umbilical cord tissue-derived mesenchymal stem cells in the treatment of multiple sclerosis. Journal of translational medicine. 2018;16(1):57.

35. Li W, Moore MJ, Vasilieva N, et al. Angiotensin-converting enzyme 2 is a functional receptor for the SARS coronavirus. Nature. 2003;426(6965):450-454.

36. Shulla A, Heald-Sargent T, Subramanya G, Zhao J, Perlman S, Gallagher T. A transmembrane serine protease is linked to the severe acute respiratory syndrome coronavirus receptor and activates virus entry. J Virol. 2011;85(2):873-882.

37. Li F, Li W, Farzan M, Harrison SC. Structure of SARS coronavirus spike receptor-binding domain complexed with receptor. Science. 2005;309(5742):1864-1868.

38. Mansouri N, Willis GR, Fernandez-Gonzalez A, et al. Mesenchymal stromal cell exosomes prevent and revert experimental pulmonary fibrosis through modulation of monocyte phenotypes. JCl Insight. 2019;4(21).

39. Mohammadipoor A, Antebi B, Batchinsky Al, Cancio LC. Therapeutic potential of products derived from mesenchymal stem/stromal cells in pulmonary disease. Respiratory Research. 2018;19(1):218.

40. Cruz FF, Rocco PRM. Stem-cell extracellular vesicles and lung repair. Stem Cell Investig. 2017;4:78.

41. Golchin A, Seyedjafari E, Ardeshirylajimi A. Mesenchymal Stem Cell Therapy for COVID-19: Present or Future. Stem Cell Rev Rep. 2020.

42. Moll G, Drzeniek N, Kamhieh-Milz J, Geissler S, Volk H-D, Reinke P. MSC Therapies for COVID-19: Importance of Patient Coagulopathy, Thromboprophylaxis, Cell Product Quality and Mode of Delivery for Treatment Safety and Efficacy. Frontiers in Immunology. 2020;11:1091.

43. Bikdeli B, Madhavan MV, Jimenez D, et al. COVID-19 and Thrombotic or Thromboembolic Disease: Implications for Prevention, Antithrombotic Therapy, and Follow-up. J Am Coll Cardiol. 2020.

44. Tang N, Li D, Wang X, Sun Z. Abnormal coagulation parameters are associated with poor prognosis in patients with novel coronavirus pneumonia. J Thromb Haemost. 2020;18(4):844-847.

45. Cui QC, C.; Huang, C.; Zhou, W.; Ji, X.; Zhang, F.; Wang, L.; Zhou, Y. AGTR2, One Possible Novel Key Gene for the Entry of 2019-nCoV into Human Cells. Preprints. 2020(2020020194). 
Figures

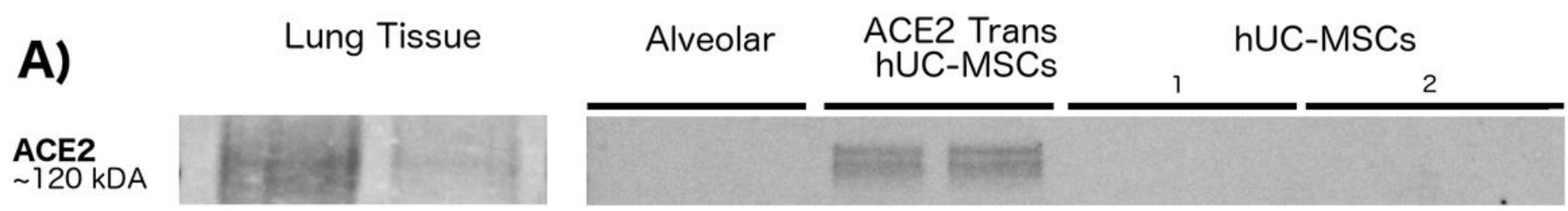

\section{TMPRSS2}

$\sim 50 \mathrm{kDA}$

\section{GAPDH}

$\sim 37 \mathrm{kDA}$
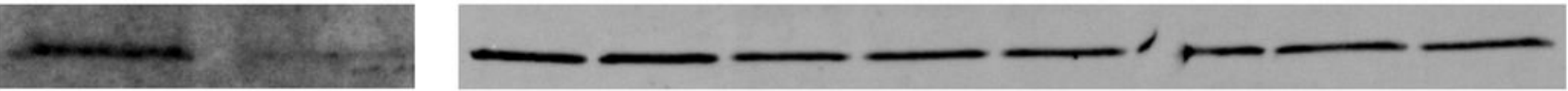

B)
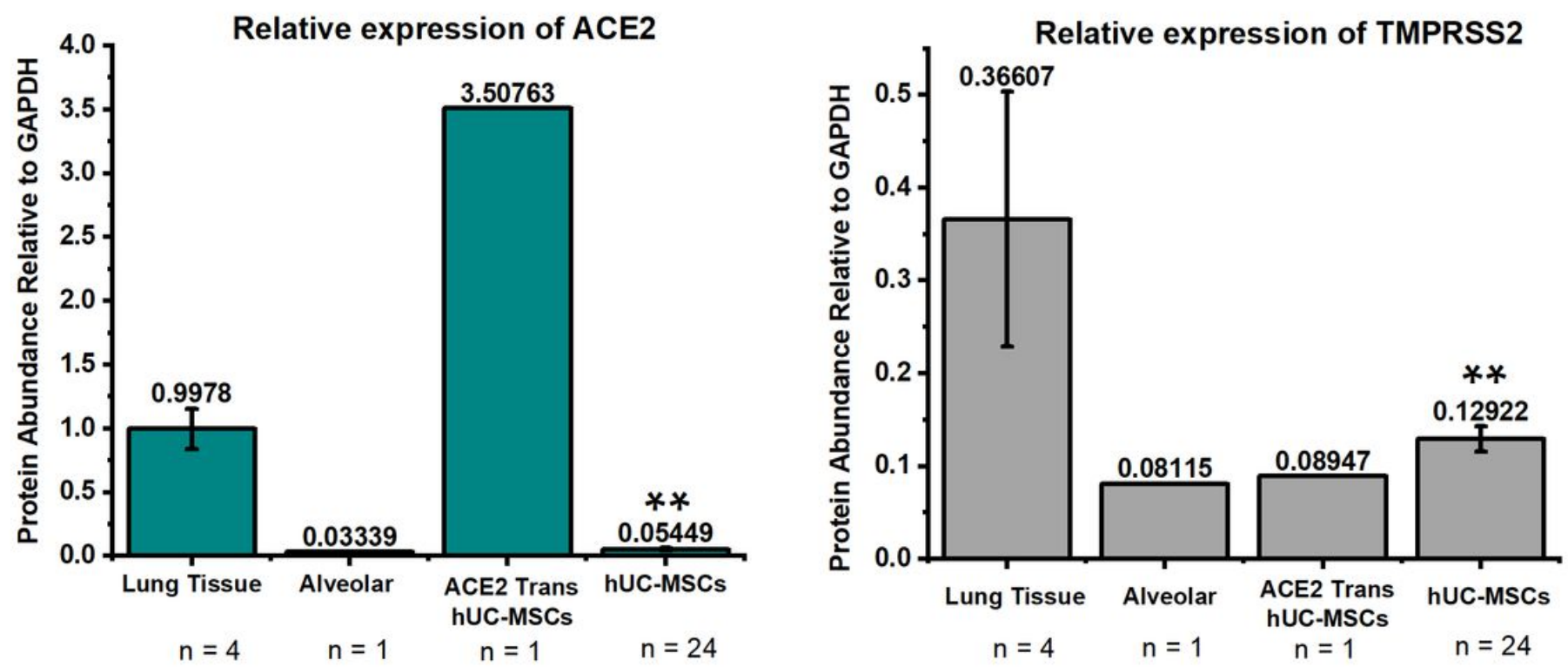

Figure 1

A. Representative blots. B. Expression levels of ACE2 and TMPRSS2 relative to GAPDH in hUC-MSCs and protein homogenates from human lung. hUC-MSCs had significantly lower expression of ACE2 ( $p=0.002)$ and TMPRSS2 ( $p=0.008)$ compared to lung homogenates. 

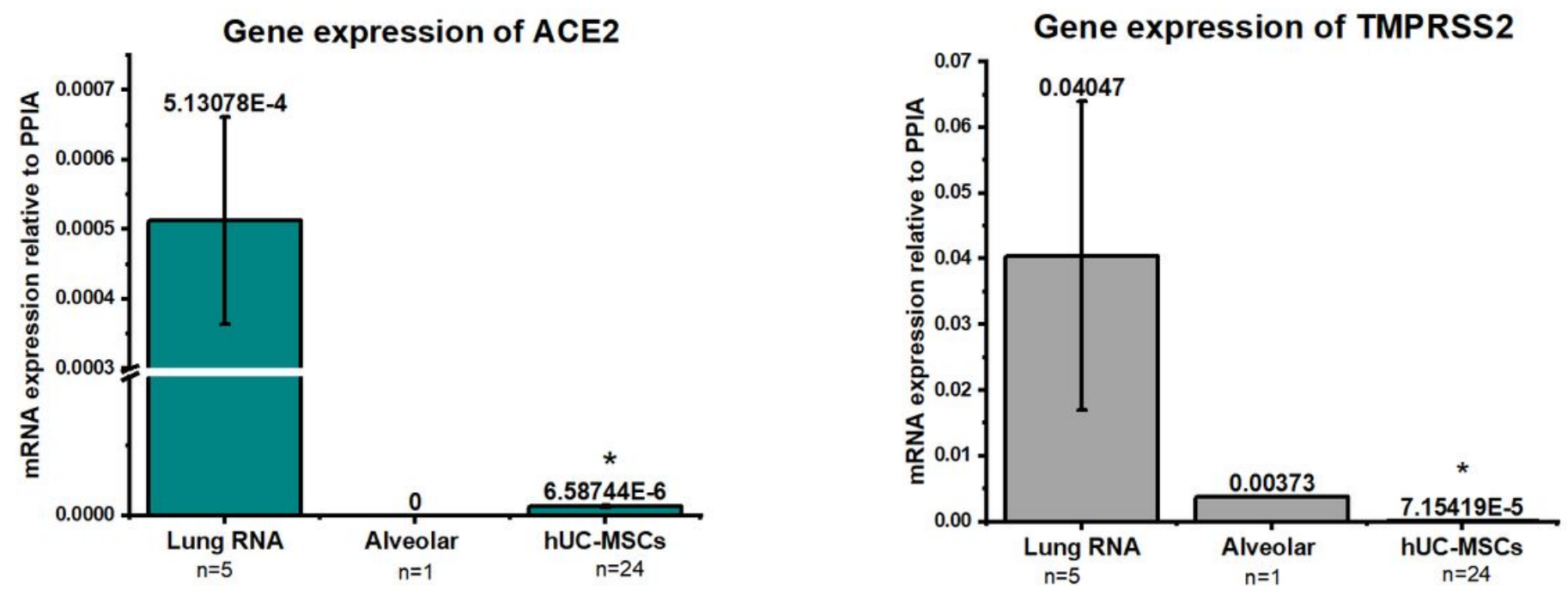

Figure 2

ACE2 and TMPRSS2 expression in human lung-derived tissue, alveolar cells, and hUC-MSCs on qPCR. The relative expression of the genes of interest was normalized to the expression of PPIA. Human lung RNA and human pulmonary alveolar cells were used as controls (tissue and single cells). The expression of ACE2 and TMPRSS2 was significantly lower in hUC-MSCs compared to human lung tissue $(P \leq 0.001$ * for both).
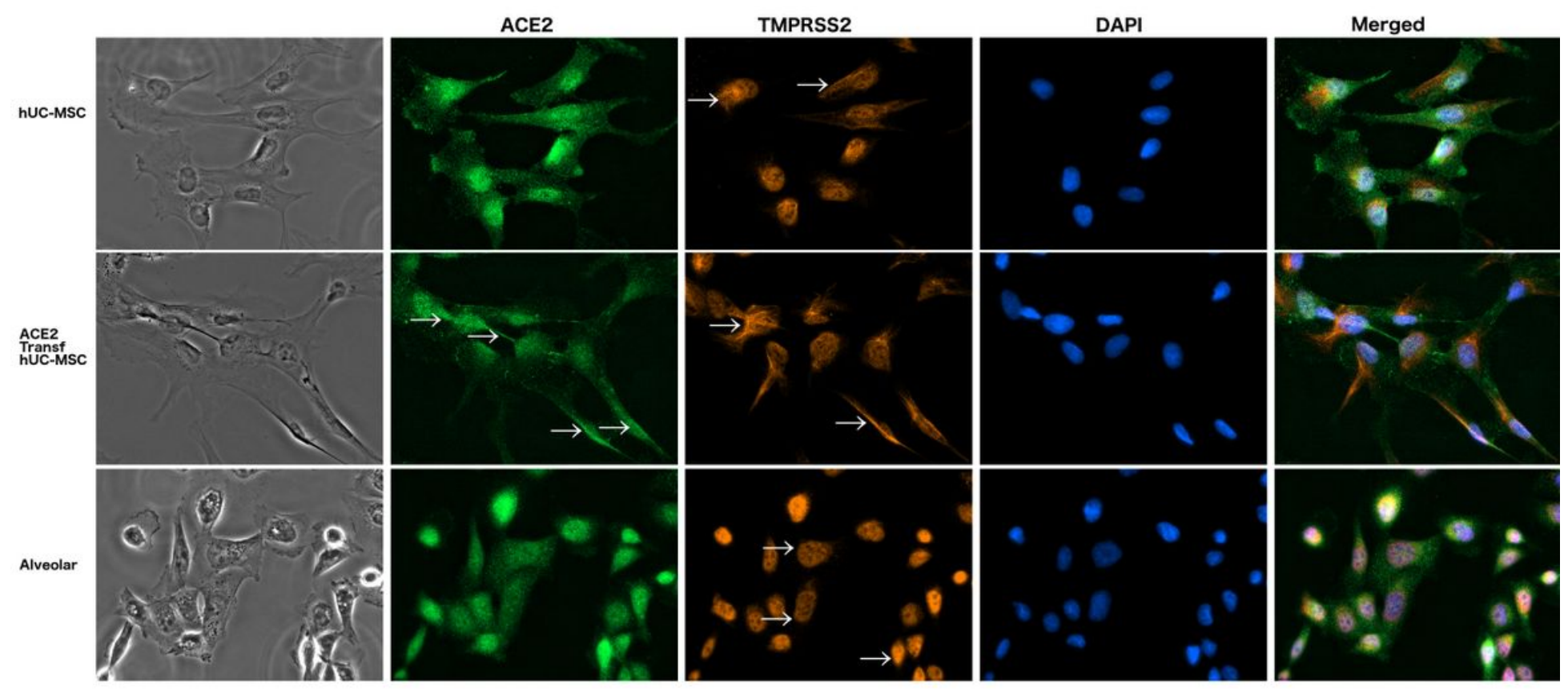

Figure 3

Immunofluorescent staining of ACE2, TMPRSS2 and nuclei for human pulmonary alveolar cells type I, hUC-MSC transfected with an ACE2 plasmid, and hUC-MSCs. White arrows indicate ACE2 or TMPRSS2 expression. 
A)

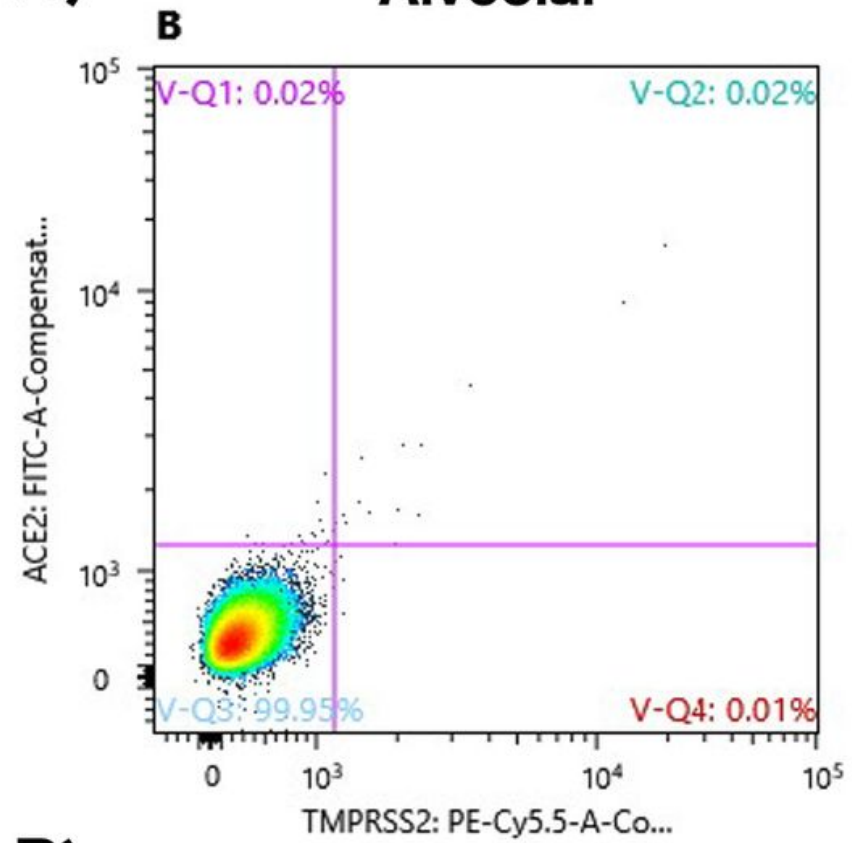

hUC-MSC

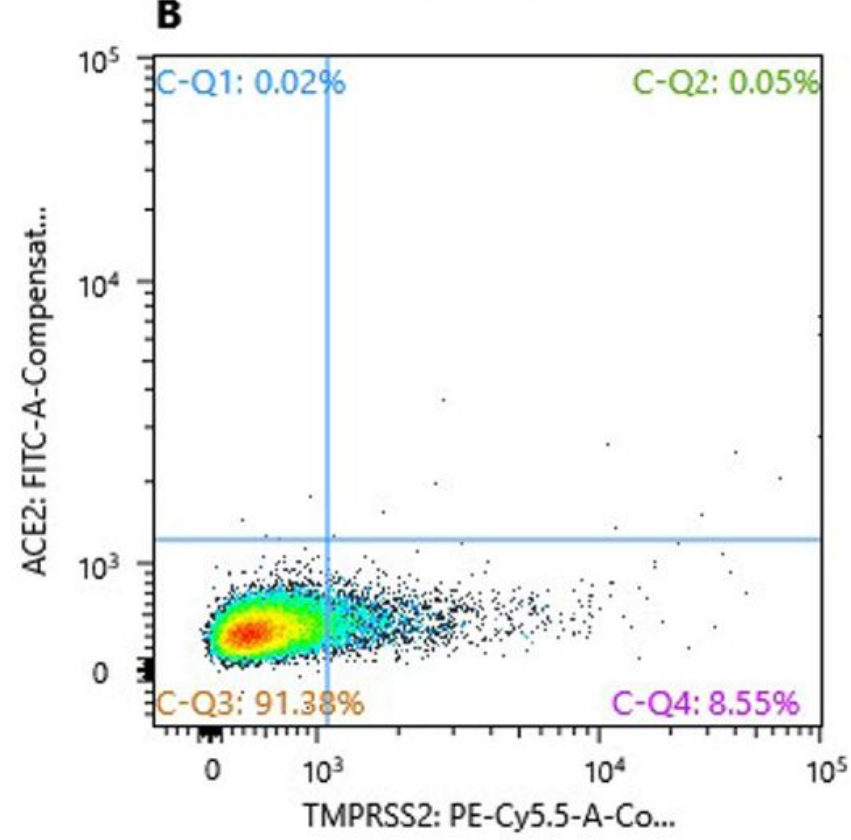

B)

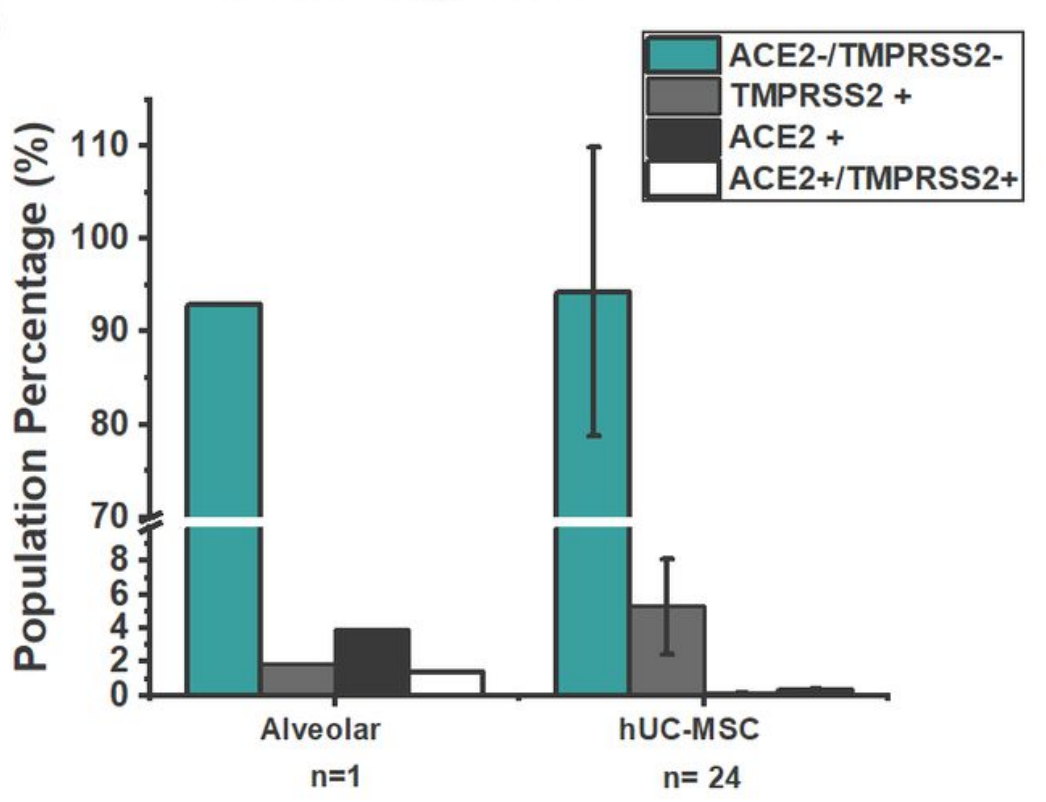

Figure 4

Flow cytometry for ACE2 and TMPRSS2 of human pulmonary alveolar cells type I, and hUC-MSC. A) Quadrant gates. The position on the quadrant relative to the quadrant gates (in blue) indicates whether cells are positive/negative for ACE2 (x-axis) or TMPRSS2 (y-axis). B) Mean of flow cytometry percentages. 


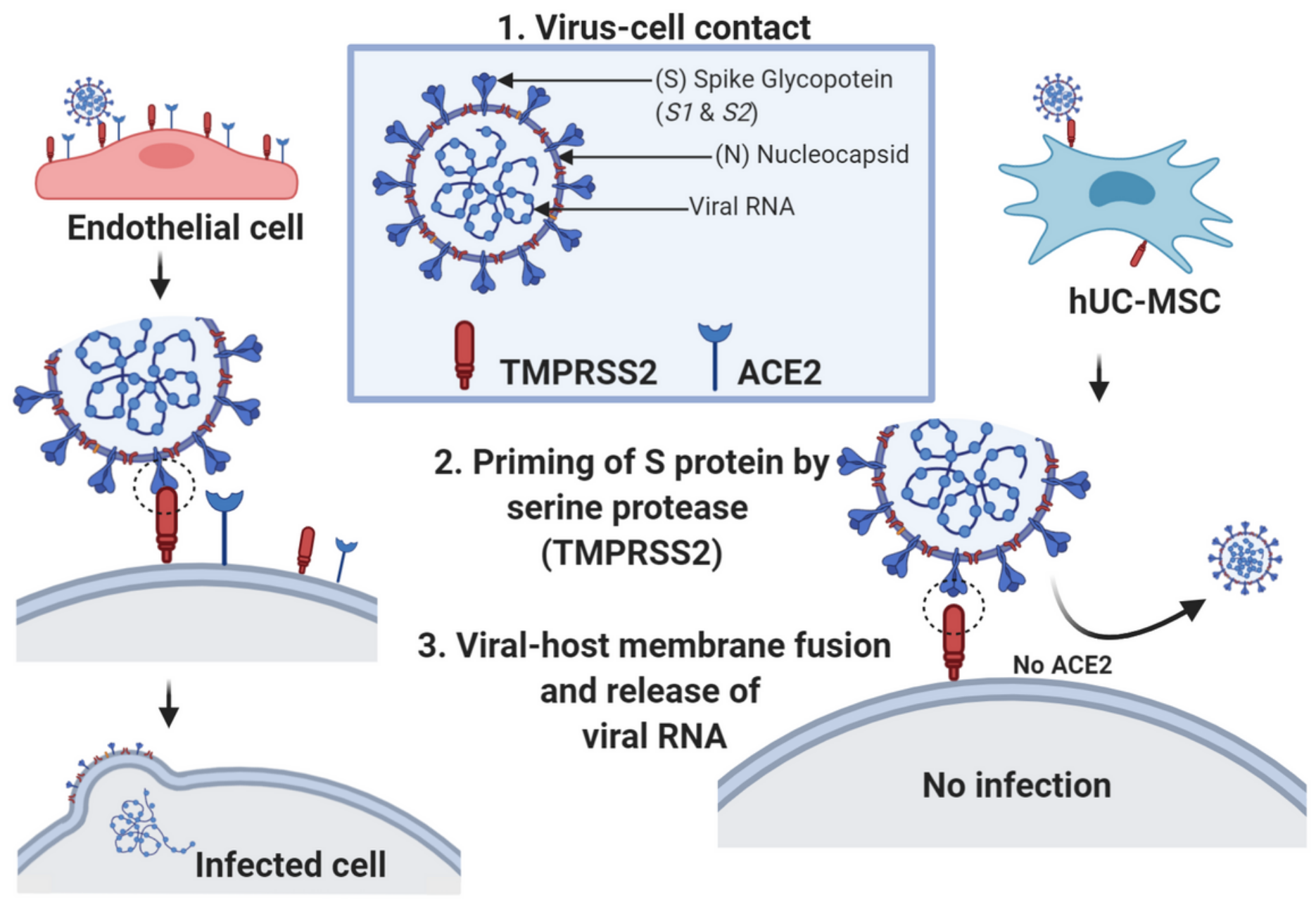

Figure 5

Left: mechanisms of infection of SARS-COV-2 in a common host cell, positively expressing ACE2 and TMPRSS2. Right: mechanisms of infection of SARS-COV-2 in a mesenchymal stem cell, negatively expressing ACE2 and TMPRSS2. Figure created with BioRender.com. 